\title{
Evaluación de dos cursos modulares en la Universidad de Costa Rica a partir de la conceptualización de la flexibilidad curricular en la educación superior ${ }^{1}$
}

\author{
Evaluation of Two Modular Courses in the University of Costa Rica based on the Curricular \\ Flexibility Conceptualization in Higher Education ${ }^{2}$
}

\section{Avaliação de dois cursos modulares da Universidade da Costa Rica com base na conceitualização da flexibilidade curricular no ensino superior ${ }^{3}$}

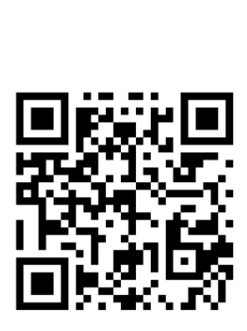

Diana Fallas-Rodríguez

Universidad de Costa Rica

San Ramón, Costa Rica

diana.fallas@ucr.ac.cr

https://orcid.org/0000-0002-7312-3680

Ismael Guido-Granados

Universidad de Costa Rica

San Ramón, Costa Rica

ismael.guido@ucr.ac.cr

https://orcid.org/0000-0001-8984-1380

Recibido • Received • Recebido: 13 / 10 / 2019

Corregido $\cdot$ Revised $\cdot$ Revisado: 07 / 03 / 2021

Aceptado • Accepted • Aprovado: 05 / 04 / 2021

\footnotetext{
${ }^{1}$ Del trabajo final de graduación para optar por el grado de Magíster en Docencia Universitaria, titulado Propuesta de mejoramiento de dos cursos modulares de la carrera Bachillerato en Gestión de los Recursos Naturales, Sede de Occidente, Universidad de Costa Rica, desde la política institucional sobre flexibilidad curricular.

${ }^{2}$ From the final work of graduation to choose the degree of Magister in University Teaching, titled Proposal for improvement of two modular courses from the Bachelor Degree in Management of Natural Resources, Sede de Occidente, University of Costa Rica, from the institutional policy on flexibility curriculum.

${ }^{3}$ Do trabalho final de graduação para optar pelo Mestrado em Ensino Universitário, denominado Proposta para melhoria de dois cursos modulares do Bacharelado em Gestão de Recursos Naturais, Sede Ocidente, Universidade da Costa Rica, da política institucional de flexibilidade curricular.
} 
http://doi.org/10.15359/ree.25-2.13

http://www.una.ac.cr/educare

educare@una.ac.cr

\begin{abstract}
Resumen:
Objetivo: Evaluar el desarrollo de las unidades didácticas Gestión de la vida silvestre y Problemas ambientales y soluciones sostenibles, de la carrera de bachillerato en Gestión de Recursos Naturales de la Universidad de Costa Rica (UCR), a partir de la conceptualización de la flexibilidad curricular en esta institución. Metodología: Se trató de una investigación cualitativa con perspectiva fenomenológica hermenéutica. La población permitió comprender el ambiente y el problema con una muestra no probabilística. La información se recopiló del personal docente de los cursos, el estudiantado matriculado, y los documentos relacionados con la normatividad en cuanto flexibilidad curricular dentro de la UCR. Las técnicas de recolección de datos fueron el análisis documental y la entrevista semiestructurada. El análisis de la información se realizó desde la triangulación hermenéutica. Resultados: No se reconoce la flexibilidad curricular desde el concepto universitario, el personal docente y estudiantes no identifican la plataforma de mediación virtual como oficial, y se presenta un enfoque positivista a criterio del personal docente; pero el estudio encontró un impulso del enfoque constructivista. Conclusiones: La flexibilidad curricular ha nacido de las mismas unidades académicas para implementar en sus actividades sistemas flexibles dentro del proceso didáctico, lo que hizo necesario normar la flexibilidad curricular, posterior al uso de módulos en la carrera. La Universidad necesita una divulgación de sus propias tendencias o concepciones al respecto de la flexibilidad, así como una reflexión o discusión de los alcances de esta dentro de la praxis pedagógica.
\end{abstract}

Palabras claves: Curso por módulos; diseño; docencia; enseñanza superior; flexibilidad curricular.

\begin{abstract}
:
Objective: To evaluate the development of the didactic units of Gestión de la vida silvestrey Problemas ambientales y soluciones sostenibles of the Baccalaureate Career in Gestión de Recursos Naturales of the Universidad de Costa Rica (Wildlife Management and Environmental Problems and Sustainable Solutions, of the bachelor's degree program of Natural Resources Management at the University of Costa Rica-UCR), based on the conceptualization of curricular flexibility in this institution. Method: A qualitative research design was implemented with a hermeneutic phenomenological perspective. The population allowed understanding the environment and the problem with a nonprobabilistic sample. The information was collected from the teachers of the courses, the enrolled students, and the documents related to the curricular flexibility in UCR regulations. The data collection techniques were documentary analysis and semi-structured interviews. The analysis of the information was made from the hermeneutical triangulation. Results: curricular flexibility is not recognized from the university concept, and teachers and students do not identify the virtual mediation platform as official, and there is a positivist approach in teachers' perspectives, but the study found a boost of the constructivist approach. Conclusions: curricular flexibility was born in the academic units themselves to implement flexible systems within didactic processes; this made it necessary to regulate the curricular flexibility after creating modular courses in the career. The University needs to disseminate its own trends or conceptions regarding flexibility, as well as a reflection or discussion on the scope of this within the pedagogical practice.
\end{abstract}

Keywords: Curricular flexibility; design; higher education; modular courses; teaching profession. 
http://doi.org/10.15359/ree.25-2.13

\begin{abstract}
Resumo:
Objetivo: Avaliar o desenvolvimento das unidades didáticas Gestão da Vida Silvestre e Problemas Ambientais e Soluções Sustentáveis do curso de Bacharelado em Gestão de Recursos Naturais da Universidade da Costa Rica (UCR) desde a ótica da conceituação de flexibilidade curricular nesta instituição. Metodologia: Trata-se de uma investigação qualitativa com perspectiva fenomenológica hermenêutica. A população nos permitiu entender o ambiente e o problema com uma amostra não probabilística. As informações foram coletadas através dos professores dos cursos, dos alunos matriculados e dos documentos relacionados aos regulamentos relativos à flexibilidade curricular na UCR. As técnicas de coleta de dados foram análise documental e entrevista semiestruturada. A análise das informações foi realizada a partir da triangulação hermenêutica. Resultados: a flexibilidade curricular não é reconhecida a partir do conceito da universidade, professores e alunos não identificam a plataforma de Mediação Virtual como oficial, e uma abordagem positivista é apresentada a critério dos professores, no entanto, o estudo encontrou um impulso na abordagem construtivista. Conclusões: a flexibilidade curricular nasceu das próprias Unidades Acadêmicas para implementar sistemas flexíveis em suas atividades no processo didático, o que tornou necessário regular a flexibilidade curricular, após o uso de módulos no curso. A Universidade precisa disseminar suas próprias tendências ou concepções sobre flexibilidade, bem como refletir ou discutir seu escopo dentro da práxis pedagógica.
\end{abstract}

Palavras-chave: Curso por módulos; design; ensino; ensino superior; flexibilidade curricular.

\title{
Introducción
}

La educación superior ha sufrido transformaciones desde fines del siglo XX, tanto en aspectos didácticos como de orden económico; los más influyentes son aquellos propuestos por entidades como el Banco Mundial (BM), la Organización de las Naciones Unidas para la Educación, la Ciencia y la Cultura (UNESCO, por sus siglas en inglés), la Organización para el Desarrollo y la Cooperación Económica (OCDE), entre otros, los cuales se han reproducido a nivel internacional y en la educación costarricense.

En una publicación de Collis y Moonen (2011), se señala que aspectos de diversa índole (políticos, sociales, filosóficos, económicos y educativos) provocaron un incremento en el interés por flexibilizar la educación superior como efecto, además, de la evolución de la tecnología y el uso del internet a finales del siglo XX. Agregan a esto la motivación de las instituciones y gobiernos para ofrecer una educación más flexible, mediante el uso de"una serie de experimentos con nuevos métodos pedagógicos y nuevas formas de interacción y recursos para el aprendizaje digital" (p. 16).

A estos elementos se sumaron la eficacia y la efectividad; perdió, en ese alcance, la eficiencia, lo cual podría afectar la calidad de la educación que se da a las futuras personas profesionales. Estas transformaciones con eficacia y efectividad han buscado estrategias para dinamizar los procesos de aprendizaje, cuyo resultado dentro de las carreras universitarias es la 
http://doi.org/10.15359/ree.25-2.13

http://www.una.ac.cr/educare

educare@una.ac.cr

flexibilidad curricular y el uso de cursos con organización académica multidisciplinar o módulos, sin que esta modalidad destaque como parte de la oferta en otros estudios de carácter similar a nivel internacional, lo cual reiteraría la posición de Collis y Moonen (2011) respecto a los experimentos con los métodos pedagógicos.

A partir de lo anterior, desde mediados de la década de 1990, en el mundo se empezaron a acuñar conceptos que pretendían ajustar las decisiones de las universidades con las tendencias que se demandaban para repensar, transformar y mejorar la oferta académica superior. Sin embargo, desde el inicio de la educación abierta mucho antes, existe una confusión entre esta, y el espacio abierto. Al respecto, el grupo Center for Open Education Research ([COER] por sus siglas en inglés,), de la Universidad de Oldenburg, en Alemania, refiere que algunos de los conceptos utilizados han sido acceso, flexibilidad, equidad, colaboración, agencia, democratización, justicia social, transparencia y eliminación de barreras (Zawacki-Richter et al, 2020).

Ante la ambigüedad que se presenta, la flexibilidad curricular ha sido una tendencia de las universidades en el mundo, pero su conceptualización responde a elementos de carácter social, cultural, histórico, político, y económico, por lo que, en Costa Rica, el análisis de las transformaciones debe enmarcarse en el escenario propio de cada universidad, así como en las prácticas que se han ligado a esa educación flexible.

Por lo tanto, esta investigación hace una inmersión en la praxis pedagógica de cursos modulares de la carrera de bachillerato en Gestión de Recursos Naturales de la Universidad de Costa Rica, con la finalidad de conocer el desarrollo de las unidades didácticas Gestión de la vida silvestre y Problemas ambientales y soluciones sostenibles, desde la flexibilidad curricular concebida por la Universidad de Costa Rica (UCR).

\section{Antecedentes}

En la historia de la educación superior, la concepción de los espacios de aprendizaje (físicos o metodológicos) ha ido variando de acuerdo con las herramientas disponibles, las características sociodemográficas y culturales de las naciones, la intencionalidad en el proceso enseñanza aprendizaje y otros intereses, unas veces de carácter pedagógico, otras relacionadas con cambios globales.

La globalización ha sido uno de los determinantes más significativos para el cambio o las transformaciones en la educación superior (Zawacki-Richter et al., 2020). En ese sentido, la apertura al pensamiento de la educación abierta, a distancia o flexible data de mediados del siglo XX, pero no es sino hasta finales de este mismo que ese ritmo de intercambio global propicia y exige, a su vez, los cambios más significativos (Collis y Moonen, 2011). 
Entre los pasos que se fueron dando alrededor del mundo, la Universidad Nacional Autónoma de México [UNAM] inició, en 1972, con el Sistema Universitario Abierto (SUA), el cual buscaba dar acceso a personas a quienes no les era posible asistir a las aulas, lo cual constituye uno de los referentes tanto históricos como del sistema de enseñanza. Ya para 1997, se fortaleció con la creación de una oficina que coordinara la educación a distancia, y en 2009, se formalizó como el Sistema Universitario Abierto y de Educación a Distancia o SUAyED. Diez años después alcanzó un aproximado de 60 programas en los diferentes niveles (pregrado, grado y posgrado) e incluso, una escuela secundaria en línea (Cervantes-Pérez et al., 2019).

A pesar de la concepción mostrada por la UNAM, los determinantes mencionados, sociales, políticos, económicos, culturales e incluso, los pedagógicos, propios de cada zona geográfica, han permitido acuñar terminología distinta, la cual hace referencia a diferentes aspectos de educación flexible, en algunos casos como sinónimos, y en otros refiriéndose a procesos distintos, íntimamente vinculados con las condiciones propias de cada país. Así, Flannery y McGarr (2014) demostraron que la bibliografía relacionada con la flexibilidad o el aprendizaje flexible se enfoca en dos discursos básicos. Por un lado, se tiene que los entornos educativos dispongan de mayor acceso y accesibilidad, mientras que, por otro, la finalidad se dirige al estudiantado, para proporcionar más opciones y control, entendido esto como flexibilidad logística y flexibilidad pedagógica.

Con estos antecedentes, la conceptualización y transformación que se dio en Costa Rica no fue diferente de lo que ocurría en el resto del mundo. En particular, las diferentes universidades estatales fueron apropiándose y construyendo sus concepciones específicas, así como métodos y formas para abordar la educación abierta, a distancia o la flexibilidad.

Por lo tanto, interesa principalmente cómo se construyó la conceptualización que se tiene en la educación superior estatal y la normativa específica dentro de la Universidad de Costa Rica, sin dejar de lado el abordaje que se ha dado desde el Consejo Nacional de Rectores (CONARE) y las universidades estatales que lo conforman.

Las universidades estatales plantean la flexibilización curricular de acuerdo con sus intereses y particularidades propias del modelo pedagógico dominante en su quehacer académico, lo cual refuerza la forma en que se ha venido dando en otros escenarios alrededor del mundo.

Específicamente en CONARE, el plan nacional de la educación superior constituye el documento en el cual se plasman todos los procesos, metas y acciones que se propone desarrollar, y marca la ruta a seguir por las universidades en todas sus áreas sustantivas (docencia, investigación y acción social). 
http://doi.org/10.15359/ree.25-2.13

http://www.una.ac.cr/educare

educare@una.ac.cr

El apartado de docencia es donde se pueden encontrar propuestas ligadas con la flexibilización curricular. El punto siete de las aspiraciones del marco estratégico en el plan nacional de la educación superior estatal (Consejo Nacional de Rectores [CONARE]. Oficina de Planificación de la Educación Superior [OPES], 2016), establece que se deberá "[promover] la renovación, el uso y la creación de nuevas tecnologías asociadas ... [a los] proyectos universitarios que procuren el beneficio social, natural y ambiental" (p. 79); donde la renovación (diferente de la innovación), el uso y la creación de tecnologías son una tarea transversal en las actividades, pues es esta última uno de los elementos con los cuales se pueden flexibilizar los programas.

El objetivo estratégico 5.5, correspondiente a la oferta académica y planes de estudio, hace referencia a la diversificación e innovación de "los planes de estudio ... con el estado del conocimiento de la época y la sociedad" (CONARE. OPES, 2016, p. 84). Lo anterior no se puede desligar del uso y creación de tecnologías, por cuanto con ellas se puede innovar en los planes (aunque la innovación va más allá de incorporar la tecnología).

En el apartado de las acciones y metas de este mismo objetivo, se procura el logro de la innovación y la flexibilidad curricular; se mencionan, incluso, los cursos bimodales o de alta virtualidad (CONARE. OPES, 2016).

En relación con las universidades estatales, la Universidad Nacional (UNA) consigna en la Gaceta extraordinaria N.o 21-2012 del 31 de octubre del 2012, las políticas y lineamientos curriculares, donde señala que la "flexibilidad curricular es una propuesta alternativa a la concepción lineal y rígida de la formación de profesionales" Universidad Nacional [UNA], Consejo Universitario [CU], (2012, p. 91). Asimismo, se señala en el mismo documento:

[Se debe] garantizar la flexibilidad curricular de los planes de estudio, promoviendo entre otros aspectos, la participación del estudiante en la definición de su currículo, la ampliación y diversificación de las opciones de formación profesional; la incorporación de competencias acordes con los cambios del entorno; la creación de cursos comunes que permitan la movilidad de los estudiantes en diferentes carreras; el fortalecimiento de cursos optativos, así como el desarrollo de investigaciones, proyectos y prácticas que enriquezcan el crecimiento personal y profesional de los estudiantes. (p. 24)

Por su parte, de acuerdo con Lira Valdivida y Fëdorov Fëdorov (2002), el InstitutoTecnológico de Costa Rica (ITCR) cuenta en el tema de la flexibilidad curricular con más de 15 años de experiencia, en especial en la pedagogía virtual, de la que se desprende el criterio de flexibilidad en la didáctica y comprensión de las variaciones en el estilo cognitivo de aprendizaje. Estos mismos estudios señalan, como una de las recomendaciones de la experiencia del aprendizaje en línea, hacer los cursos modularmente para tener más flexibilidad y compartir los módulos en diferentes cursos o programas complementarios (Lira Valdivida y Fëdorov Fëdorov, 2002). 
En cuanto a la Universidad Estatal a Distancia (UNED), se tiene como referencia que desde sus orígenes y por su razón de ser, es la que ha presentado mayor incidencia en la flexibilidad curricular, en especial en las variables relacionadas con la educación a distancia, no así en la existencia de cursos modulares. En la actualidad, la UNED ha hecho énfasis en el empleo de las tecnologías modernas de información ( $\mathrm{TI}$ ) lo que se considera como la tercera generación de educación a distancia (Hidalgo, 2001).

Finalmente, en el caso de la más reciente de las universidades estatales, la Universidad Técnica Nacional (UTN), la flexibilidad curricular se ha dado mediante el uso de las tecnologías de la información y la comunicación (TIC), las cuales son vistas como un "medio de apoyo para la implementación de metodologías diversificadas en los ambientes de aprendizaje, siendo estos presenciales, bimodales y/o virtuales" (Camacho Zúñiga et al., 2016, p. 1).

Todas las instituciones estatales de educación superior están comprometidas de una u otra manera con la tecnología como parte de los elementos de una propuesta de flexibilidad curricular. Principalmente, se considera, dentro de esta la renovación de los planes (CONARE), la autonomía de las personas estudiantes, las competencias y los cursos comunes a varias carreras (UNA), y la pedagogía virtual y los cursos modulares (TEC).

Por otra parte, en los antecedentes relacionados con los aspectos normativos, el caso de la UNAM muestra cómo se han dado los cambios en este sentido, para lo cual se deben generar elementos reglamentarios o directrices, que permitan el desarrollo de iniciativas o acciones en la enseñanza a través de la flexibilidad curricular (Cervantes-Pérez et al., 2019).

En términos generales, las instituciones han introducido cambios paulatinos de acuerdo con las necesidades que demanda la educación superior, los gobiernos, o ambos, desde los contextos particulares y globales. Palmer (2011) reafirma que el llamado a la flexibilidad surge como respuesta a una serie de necesidades de una variedad de partes interesadas, en diferentes momentos y en muchos contextos. Otro ejemplo al respecto es que, en el año 2011, el gobierno de Irlanda estableció la estrategia de la educación superior para 2030, en la cual se demanda que el sistema debe incluir más programas de aprendizaje flexibles, más rutas flexibles para el avance y la transferencia, y acuerdos de trabajo más flexibles (Flannery y McGarr, 2014, p. 419).

En el caso particular de la Universidad de Costa Rica, puede ser de los más representativos para establecer el estado de la cuestión en la investigación a nivel local, e identificar las concepciones que, respecto de la flexibilidad curricular, se han promovido, tanto con resoluciones de la Vicerrectoría de Docencia (responsable de la administración curricular universitaria y docente), así como del Centro de Evaluación Académica (CEA), instancia que apoya la construcción y desarrollo de planes de estudio. 
http://doi.org/10.15359/ree.25-2.13

http://www.una.ac.cr/educare

educare@una.ac.cr

De acuerdo con estos niveles, en la política universitaria para el 2016-2020, en su eje de excelencia académica, se estipula para el alcance del propósito "flexibilizando la estructura y gestión de los planes de estudio tanto de grado como de posgrado, sobre la base de una visión crítica, humanista, inclusiva, de derechos humanos y de protección del ambiente" (Universidad de Costa Rica [UCR], Consejo Universitario [CU], 2015, p. 2).

Estas políticas fueron concebidas en el 2015, por lo que se mantiene la congruencia en las acciones universitarias dentro de las resoluciones de la Vicerrectoría de Docencia (VD) como responsable de las labores necesarias para la excelencia académica, con la resolución VD-R9374-2016, donde se define la flexibilidad curricular como "una manera de facilitar al personal docente la incorporación de modalidades innovadoras de docencia, y al estudiantado el avance en su carrera" (Universidad de Costa Rica [UCR], Vicerrectoría de Docencia [VD], 2016a, p. 74).

En el plano curricular, ello implica la posibilidad de plantear e implementar planes de estudio y cursos que puedan adaptarse o modificarse, según las necesidades e intereses del estudiantado, las necesidades y problemas de la sociedad, y los niveles de desarrollo de la respectiva área de conocimiento. (UCR, VD, 2016a, p. 74)

También se puede percibir en este documento que una docencia "innovadora y flexible, que fomente el aprendizaje colaborativo [por medio de diversos entornos, ámbitos o metodologías]" (UCR, VD, 2016a, p. 74) es parte de los aspectos que se deben buscar para la flexibilidad curricular y queda a criterio de las unidades académicas hacerlo.

Sin embargo, en el diseño de planes de estudio o en revisiones de estos, la flexibilización está más relacionada con las tendencias universitarias que con un análisis del plan. Esto promueve modificaciones en elementos sin dominio de su conceptualización, que al implementarse resultan en diseños pedagógicos tradicionalistas, muy alejados de la flexibilización.

Otra de las resoluciones de la VD propone que los "vertiginosos cambios culturales, científicos y tecnológicos característicos del mundo actual en todas las esferas de la actividad humana, exigen definir estrategias y tomar acciones para la innovación y el cambio de paradigmas en la Educación Superior" (UCR, VD, 2016b, p. 1), ya que los "procesos de aprendizaje en diversos espacios en la Educación Superior, trascienden la visión compartimentada y las fronteras disciplinarias, de ahí la necesidad de promover una mayor flexibilización curricular en el diseño de planes de estudio" (UCR, VD, 2016b, p. 4).

Finalmente, en la revisión de estos antecedentes, la propuesta de creación de la carrera de Gestión de los Recursos Naturales no presenta ninguno de los elementos que se puedan concebir como flexibilización curricular, como los propósitos, las modalidades de trabajo (virtual o presencial), horarios, requisitos de los cursos, entre otros. Esto parece evidenciar que hay una incongruencia entre la concepción y el uso de cursos modulares como parte del plan de estudios.

8 
http://doi.org/10.15359/ree.25-2.13

\section{Referentes conceptuales}

Los cambios a nivel económico en el mundo se han filtrado en la educación superior, sin posibilidad de obviarlos. Por ello, las transformaciones que viven las universidades deben ser parte de una reflexión, tanto del colectivo académico, como del estudiantado y personas administrativas. En este sentido, Pedroza Flores y García Briceño (2005) proponen:

La flexibilidad académica y curricular es un tema en la agenda de discusión actual en las IES [instituciones de educación superior], a partir de los cambios emprendidos con la modernización educativa, tendientes a superar las debilidades que se registran en el modelo académico rígido. (p. 13)

Las discusiones cuyo bordaje es necesario en los escenarios universitarios requieren reconocer que el conocimiento científico se ha vuelto para la economía un bien intercambiable. Así, la flexibilidad curricular es una estrategia para promover modelos educativos ajustados a las necesidades de formación (de la industria o mercado), y responder no únicamente a las eventualidades de los sistemas económicos, sino a las realidades históricas, culturales y sociales de cada país.

Los cambios se han estimulado desde distintos organismos internacionales como la OCDE, UNESCO y el BM, a través de diversos ajustes:

Organización académica multidisciplinaria (sistemas modulares), profesionalización de la carrera docente (programas de estímulos a la productividad y calidad), sistemas flexibles (educación no presencial y uso intensivo de recursos tecnológicos), curriculum integral (áreas básicas y materias optativas) y crecimiento regulado (desconcentración y descentralización de la oferta educativa). (Pedroza Flores y García Briceño, 2005, p. 20)

Estos aires de cambio han conceptualizado la flexibilidad curricular en el movimiento global educativo como "el proceso de intercomunicación disciplinaria orientado a facilitar la movilidad de los actores académicos, acelerar los flujos de comunicación, conectar el conocimiento con la acción y democratizar la regulación del trabajo académico" (Pedroza Flores y García Briceño, 2005, p. 34).

Sin embargo, los conceptos varían entre los países, así como los elementos que se consideran parte de la flexibilidad curricular o educación flexible. En Australia, el término general utilizado es educación flexible, e incorpora, dentro de su comprensión, el aprendizaje flexible, la enseñanza flexible y otros términos relacionados. Como resultado no existe una definición universalmente aceptada para la educación flexible. Además, se estableció que la flexibilidad puede dirigirse a diferentes aspectos en el proceso de enseñanza aprendizaje, los cuales tienen que ver con el tiempo, el ritmo, el lugar, el contenido, el estilo de aprendizaje, la evaluación, en la colaboración y en la dotación de personal (Palmer, 2011). 
http://doi.org/10.15359/ree.25-2.13

http://www.una.ac.cr/educare

educare@una.ac.cr

Para Alemania, la terminología se transforma en aprendizaje abierto, aprendizaje a distancia y educación a distancia, a menudo con un uso indistinto, y con el consenso sobre su combinación, a pesar de no ser sinónimos. En este caso, el aprendizaje abierto generalmente se refiere a la flexibilidad, el acceso, cuándo, a qué ritmo, dónde y cómo aprenden las personas (Zawacki-Richter et al., 2020).

Específicamente para la Universidad de Costa Rica, de acuerdo con la Resolución VD-R9374-2016 denominada Marco de Referencia para el Desarrollo de la Docencia en Entornos Virtuales, la flexibilidad curricular es entendida como "una manera de facilitar al personal docente la incorporación de modalidades innovadoras de docencia, y al estudiantado el avance en su carrera" (UCR, VD, 2016a, p. 74).

De esta manera, la flexibilidad curricular concede a las unidades académicas la posibilidad de sugerir y aplicar "estructuras y procesos de enseñanza y aprendizaje flexibles, en los que se trascienda los límites tradicionales de la docencia, y se creen nuevas y diversas oportunidades de formación, que incidan significativamente en los procesos educativos de la Universidad" (UCR, VD, 2016a, p. 74 2).

Otro aspecto presente en la UCR es la implementación de cursos bimodales, con algún grado de virtualidad, por medio de la plataforma conocida como Mediación virtual o aulas virtuales; resultan, entonces, las acciones mencionadas, parte de las características con que se concibe la flexibilidad.

A pesar de la existencia de las propuestas para flexibilizar el currículo e incorporar la implementación de, por ejemplo, las tecnologías de la información y comunicación (TIC) en los cursos o en algunos de ellos, el avance que se ha logrado en la UCR es limitado y de momento se circunscribe a la aplicación de la bimodalidad mediante la plataforma mencionada, con la cual se procura que las "unidades académicas desarrollen cursos con algún grado de virtualidad, en procura de una mayor flexibilidad curricular" (UCR, VD, 2016a, p. 74).

En la flexibilización de los currículos, Mirriahi et al. (2015) señalan que este debe ser conceptualizado antes de evaluar su aplicación en una propuesta de plan de estudios en educación superior, ya que puede entenderse como la flexibilización en cuanto requisitos y correquisitos, sincronía y asincronía en las sesiones, entre otros.

En la experiencia puntual de la carrera de Bachillerato en Gestión de Recursos Naturales (GERENA), esa flexibilización del currículo se circunscribe al empleo de módulos, los cuales según García Fallas et al. (2014) consiste en lo siguiente: 
http://doi.org/10.15359/ree.25-2.13

Una propuesta organizada de los elementos o componentes instructivos para que el estudiantado desarrolle experiencias de aprendizaje en torno a un determinado tema o situación problematizadora; forma parte de un diseño curricular no tradicional, donde el proceso de enseñanza y aprendizaje de una disciplina o profesión está organizado de manera tal que toda la formación responde a las necesidades reales del contexto en que se desenvuelven los profesionales. ... Organizan la experiencia del proceso de enseñanza y aprendizaje, de manera que se integran experiencias, destrezas, conocimientos, actitudes y valores en torno al eje temático específico, generalmente un eje problematizador. (pp. 71-72)

Asimismo, Clavijo y Bocanumen Gutiérrez (2006) señalan:

[Aplicados al aprendizaje, los módulos] crean un nuevo paradigma que producirá una revolución en la educación y que se puede describir a través de los siguientes cambios:

- De la instrucción, a la construcción y el descubrimiento.

- De la educación centrada en el maestro, a la educación centrada en el estudiante.

- Del aprendizaje en la escuela o la universidad, al aprendizaje durante toda su vida.

- Del aprendizaje colectivo y masificante, al aprendizaje personalizado.

- Del aprendizaje como obligación desagradable, al aprendizaje como diversión. (pp. 80-81)

El currículo del bachillerato en GERENA, el cual pretendió en su diseño ser flexible (con la introducción de cursos modulares), para ajustarse a las necesidades de la población meta y de la sociedad misma, no puede olvidar que "el currículo es un proceso social" (Jiménez Castro, 2008, p. 63), y para su construcción se requiere más que una serie de contenidos con un orden de transferencia lógico. Este refleja no solo la participación del personal docente como medio de transferencia de conocimiento, sino además el papel del estudiantado como participante activo de la enseñanza, para lo cual se deben tener presente las "dimensiones cognitiva, afectiva, social y psicomotriz" (Jiménez Castro, 2008, p. 70).

Bautista et al. (2006) hacen un planteamiento muy interesante con respecto a esta construcción social, por cuanto mencionan que en los enfoques curriculares se debe "entender e incorporar... la premisa relacionada con el hecho de que no se trata solo de enseñar, sino también hacer que los estudiantes aprendan" (p. 22). Y es que, al reflexionar sobre esto, no se puede obviar que, para lograrlo, lo que se lleva a los cursos debe ser significativo para el estudiantado, así como tener un propósito dentro de sus expectativas. Sin embargo, esto no se logra sin el reconocimiento de este como actor y no como espectador.

No es posible seguir con la estrategia a prueba de fallos, de la que habla Tourón y Campión (2013), en la cual se parte del hecho de que todo el estudiantado tiene el mismo nivel de conocimientos, destrezas y competencias; por cuanto sería negar el proceso social de currículo. 
http://doi.org/10.15359/ree.25-2.13

http://www.una.ac.cr/educare

educare@una.ac.cr

Aunque las diferencias individuales se pueden nutrir a partir del uso de tecnología, ciertamente la flexibilización curricular y el uso específico de módulos debe conceptualizarse, claramente, dentro de la propuesta de un plan de estudios, en aras de que su puesta en práctica alcance los objetivos establecidos, y no resulte en modelos pedagógicos tradicionalistas con toques de bien enajenable.

\section{Metodología}

Para el trabajo final de graduación de la Maestría en Docencia Universitaria, titulado Propuesta de mejoramiento de dos cursos modulares de la carrera Bachillerato en Gestión de los Recursos Naturales, Sede de Occidente, Universidad de Costa Rica, desde la política institucional sobre flexibilidad curricular, se usó el enfoque de investigación de tipo cualitativo a partir de un paradigma naturalista (naturalista-humanista o interpretativo) con perspectiva fenomenológica hermenéutica, ya que pretendió describir, comprender e interpretar el fenómeno desde las experiencias de los sujetos participantes. La hermenéutica permitió comprender las vivencias de la población estudiada, mediante el contraste de estas, y buscar la comprensión en los procesos de flexibilización curricular, los cuales se desarrollan en la carrera GERENA, específicamente en dos cursos modulares. Conocer, valorar y transformar se hizo desde el interior de la relación sujeto-objeto, donde el sujeto (estudiantes, docentes e investigadores) es partícipe del fenómeno estudiado (flexibilidad curricular).

En cuanto a la población de estudio, lo más importante en las indagaciones cualitativas es que los casos seleccionados como muestras o fracciones de la población permitan comprender el ambiente y el problema de investigación, aunque no sean representativas de la totalidad, por lo que se conocen también como no probabilísticas o dirigidas. Por ello, en tanto la población participante permita conocer los dos aspectos (de ambiente y el problema), los resultados del estudio tendrán validez.

Se utilizaron criterios para la selección de participantes, tanto en el caso de la población estudiantil como docente. Para ser elegible en el estudio, reconociendo como principal característica que sea población es partícipe del fenómeno, la totalidad de participantes de la muestra no probabilística debía ser población estudiantil matriculada y docentes de los cursos, al momento del estudio.

La información se recopiló de tres fuentes. Primero, la totalidad del personal docente de los cursos Gestión de vida silvestre y Problemas ambientales y soluciones sostenibles de la carrera GERENA, impartidos en el I ciclo 2017. Segundo, el estudiantado matriculado en los módulos y presentes en la clase al momento de la aplicación de los instrumentos, donde se entrevistó a 10 personas por curso. Tercero, los documentos relacionados con la normatividad en cuanto flexibilidad curricular dentro de la UCR, para lograr la triangulación de los datos. 
Las categorías de análisis en el estudio fueron la flexibilidad curricular desde la política institucional (con las subcategorías resoluciones, circulares y reglamentos de docencia), elementos de la flexibilidad curricular en los cursos modulares de la carrera GERENA (subcategorías componentes curriculares del programa, metodología y evaluación continua de los aprendizajes) y, por último, flexibilidad curricular desde la praxis pedagógica (para la cual se definieron subcategorías de entornos virtuales, herramientas en línea por estamentos, porcentajes de virtualidad, asistencia, estrategias didácticas y modalidades de evaluación).

Dado que en los enfoques cualitativos la recolección de datos no tiene como propósito medir variables, sino obtener datos, para la primera y segunda categoría se utilizó la técnica de análisis documental. En las fuentes de información, el instrumento fue una guía de preguntas abiertas y cerradas mientras que, para los elementos de la flexibilidad, se pretendió visualizar los distintos componentes a través de una lista de cotejo, con observaciones que permitieran al equipo investigador conocer la forma en la que se visualiza o evidencia el concepto de flexibilidad.

En la praxis pedagógica, se empleó la técnica de entrevista semiestructurada con preguntas generadoras, la cual se aplicó tanto al profesorado como al estudiantado (con instrumentos distintos en cada población), para entender un fenómeno a partir de la interpretación de sus participantes, donde la observación no participante podría dejar de percibir algunos elementos relevantes. Además, por medio de la entrevista se esclarece la experiencia humana subjetiva. Los instrumentos fueron validados previamente a su aplicación, con el fin de corroborar la pertinencia de las categorías y subcategorías de análisis, según los objetivos de la investigación.

De acuerdo con el componente de modalidades innovadoras de docencia en la flexibilidad curricular, el presente estudio se enfocó en esto a partir de las plataformas virtuales, estrategias metodológicas y estrategias de evaluación; sin tomar en cuenta elementos que pudieran vincularse con el avance en la carrera.

Para el análisis de la información cualitativa, el procesamiento se realizó desde la triangulación hermenéutica, la cual consiste, según Cisterna Cabrera (2005, p. 68), en "la acción de reunión y cruce dialéctico de toda la información". Desde esta perspectiva, el mismo autor plantea que los pasos para efectuar este procedimiento contemplan la selección de la información obtenida para la triangulación, cruzar la información con todos los estamentos, triangular con los datos obtenidos mediante los instrumentos $y$, finalmente, con el marco teórico. Para el estudio en particular, la triangulación hermenéutica siguió los pasos señalados por Cisterna Cabrera (2005).

\section{Resultados, análisis y discusión}

Los resultados se establecieron a partir de la información obtenida con la técnica documental y de entrevista, donde la guía de preguntas y la lista de cotejo, permitieron identificar 
http://doi.org/10.15359/ree.25-2.13

http://www.una.ac.cr/educare

educare@una.ac.cr

la política institucional desde la flexibilidad curricular, los elementos de la flexibilidad curricular en los cursos modulares y la realidad de la flexibilidad curricular aplicada. La observación participante de los investigadores, permitió vivenciar el fenómeno en estudio. Todos los hallazgos se encontraron dentro de las categorías y subcategorías de análisis, gracias a la previa validación de los instrumentos, por lo que no se obtuvo categorías emergentes.

En los resultados de la política institucional, se encontró tres resoluciones vinculadas de alguna manera con la flexibilidad curricular. La primera busca la promoción del acceso y uso democrático, la apropiación de una docencia mediada con TIC, así como una mayor seguridad técnica, informática y de gestión mediante el uso de la plataforma conocida como Mediación Virtual. No introduce ningún concepto de flexibilidad curricular, aunque propone elementos que forman parte de ella, como acelerar los flujos de comunicación, en este caso, a través de la mediación virtual.

El concepto de flexibilidad curricular se oficializó en el 2016, mediante la resolución VDR-9374-2016 Marco de referencia para el desarrollo de la docencia en entornos virtuales en la Universidad de Costa Rica, que establece la flexibilidad curricular y reafirma el uso obligatorio de la plataforma.

Esta norma deja de lado aspectos tan importantes como la movilidad entre actores académicos, "conectar el conocimiento con la acción y democratizar la regulación del trabajo académico" (Pedroza Flores, 2004, p. 10); por lo que, a pesar de establecer un concepto, se queda solamente en acelerar los flujos de comunicación.

En el mismo año que la resolución anterior, se dio la VD-R-9391-2016, titulada Creación del espacio de docencia multi-, inter-y transdisciplinario (EDOMIT). Con ella, se instaura el espacio universitario para el "fomento, creación y, si así fuera requerido, la acogida de programas, planes de estudio, proyectos de docencia, cursos y otras actividades docentes ... [de] carácter multi-, inter- y transdisciplinario" (UCR, VD, 2016b, p. 3).

Específicamente en el caso de Gestión de la Vida Silvestre, los hallazgos muestran que dos docentes utilizan la modalidad de bajo virtual (25\%), y solamente uno de ellos no la utiliza del todo. Asimismo, usan herramientas como el correo electrónico, el WhatsApp, y el Dropbox para el intercambio de información o la comunicación con el estudiantado. Sin embargo, solo uno de ellos se refirió a Mediación Virtual como plataforma institucional.

En el módulo Problemas ambientales y soluciones sostenibles solo un docente manifiesta el uso del aula virtual, pero de igual manera se refiere a las mismas herramientas del profesorado anterior. No se hace referencia a la existencia de una plataforma institucional, por lo que la mediación virtual parecen estarla relacionando con otras herramientas. 
http://doi.org/10.15359/ree.25-2.13

No se encuentra evidencia de la comprensión de la flexibilidad curricular o un proceso de sensibilización por parte de la Universidad de Costa Rica, desde donde se pueda aplicar el sentido real de la flexibilidad.

Por su parte, el estudiantado coincide con lo indicado por el personal docente en cuanto al uso de herramientas virtuales, sin ser la plataforma oficial, y se nota un desconocimiento tanto en normativa como en conceptualización. Solo se acierta desde el punto de vista de discentes, que la flexibilidad se puede plantear en adaptaciones o modificaciones de acuerdo con sus necesidades.

Para los elementos de la flexibilidad curricular en los cursos modulares, el módulo Gestión de la vida silvestre presenta varios aspectos como el mismo hecho de ser modular o las prácticas metodológicas. No obstante, en la evaluación se siguen algunos detalles más tradicionales como los exámenes y otros más innovadores, los cuales pueden ser la confección de videos o una museografía, desde una perspectiva de aprendizaje significativo o por experiencias.

Asimismo, se emplean las clases magistrales, la resolución de problemas, el trabajo colaborativo y la investigación, pero también la proyección de videos, las giras de campo, estudios de caso, historias de vida y talleres. En referencia a los aspectos evaluativos empleados, resaltan las formas tradicionales como lo es el examen y el quiz, pero también se presenta la elaboración de informes, el desarrollo de proyectos, bitácoras, videos, así como la ejecución de exposiciones.

Respecto al módulo Problemas ambientales y soluciones sostenibles el programa del curso establece que las estrategias metodológicas son parcialmente de apropiación teórica y parcialmente aplicación de problemas. Además, en la evaluación, esta es totalmente sumativa con una parcialidad de estrategias innovadoras como las mencionadas anteriormente.

No se contempla la evaluación formativa o la diagnóstica, es evidente que se centra también en modelos más conductuales donde prima la evaluación sumativa y se queda, entonces, en métodos de evaluación parcialmente tradicionales y parcialmente innovadores.

A partir de estos hallazgos se encuentra la congruencia con los lineamientos conceptuales de la flexibilidad curricular y otros contextos socioeducativos. La flexibilidad curricular, el aprendizaje flexible, la educación abierta y otros términos que se le han dado desde mediados del siglo XX muestran que no se ha llegado a un consenso en el uso y aplicación de la terminología, las técnicas o componentes de la flexibilidad, ni la etiología de su implementación.

Costa Rica ha seguido las tendencias a nivel mundial. Otros estudios sobre el aprendizaje flexible o en este caso, flexibilidad curricular, muestran que es un proceso complejo, que resulta como respuesta a necesidades de la educación superior en el mundo, pero también considera las particularidades de cada espacio geográfico (Cervantes-Pérez et al., 2019; Collis y Moonen, 2011; Flannery y McGarr, 2014; Palmer, 2011; Zawacki-Richter et al, 2020). 
http://doi.org/10.15359/ree.25-2.13

http://www.una.ac.cr/educare

educare@una.ac.cr

El concepto de flexibilidad establecido por la resolución (VD-R-9374-2016) en la Universidad de Costa Rica permanece en la teoría, pues en la praxis y en el planteamiento de los programas persiste un enfoque más positivista; los programas de los cursos no son capaces de reflejar las metodologías o la evaluación, acordes con esas posibilidades de innovación, aunque en la praxis sí lo desarrollen. Esto coincide con lo expuesto por Collis y Moonen (2011), quienes señalaron que los cambios que se realizaban para la flexibilidad parecían dividirse entre aquellos de carácter logístico y otros pedagógicos, donde los primeros resultaban los más notorios. Sin embargo, los de carácter pedagógico, y que representan tanto al estudiantado como al profesorado, quedaban ocultos entre estas transformaciones.

Por otra parte, los resultados mostraron que la perspectiva del estudiantado fue congruente con lo manifestado por el personal docente tanto en metodologías como en evaluación, pero la gama amplia en las estrategias que pueden considerarse como modalidades innovadoras no se reconocen como flexibilidad desde el concepto universitario, pues ambos estamentos desconocen la normativa y, por tanto, la definición. El hallazgo evidencia que los cambios no son percibidos como parte del fenómeno en estudio, quedan ocultos los aportes que se pueden generar en la educación superior, aun cuando la docencia y el aprendizaje consideran partícipes activos al personal docente, la población estudiantil y la pedagogía, en especial las actividades emergentes (Collins y Moonen, 2011).

Con los resultados de esta investigación es posible determinar que las unidades académicas han logrado la trascendencia a una docencia innovadora, buscando la intercomunicación que no se señala en la definición institucional (de la UCR), pero que se ha ido construyendo de manera colectiva, a partir de las necesidades y realidades las cuales surgen en los contextos de la educación superior actual.

Por esas razones, en la carrera GERENA, se ha encontrado, en la praxis, modelos más flexibles, con un impulso del enfoque constructivista, el cual se promueve desde organismos internacionales como el BM o la UNESCO, y los cambios responden, entonces, a las tendencias mundiales. Sus estrategias metodológicas coinciden con este enfoque, basado en la resolución de problemas, el aprendizaje significativo y el desarrollo de habilidades y destrezas.

Es relevante señalar que la flexibilidad curricular no ha tenido una trayectoria vertical descendente, desde lo internacional hacia lo nacional dentro de las universidades, y de estas hacia las unidades académicas. Han sido las mismas escuelas y sedes las que han partido de lo internacional para implementar en sus actividades sistemas flexibles dentro del proceso didáctico; y es a partir de esta secuencia, que se ha permitido que la Universidad atendiera la necesidad de normar la flexibilidad curricular, y se explica así que primero se diera este tipo de cambios dentro de la carrera GERENA (creada en 2009), y luego la publicación e instauración de una política universitaria al respecto, por medio de la resolución Marco de referencia para el desarrollo de la docencia en entornos virtuales (en el año 2016). 
http://doi.org/10.15359/ree.25-2.13

Se requiere que las autoridades universitarias divulguen los alcances que con la norma se pretenden, para tener currículos más flexibles y adaptados a los cambios de la educación superior, siempre atentos al contexto nacional y al compromiso con la sociedad que deben guardar las universidades estatales.

Finalmente, aunque la introducción de los módulos dentro de la carrera GERENA no ha sido sincronizada con la norma universitaria de flexibilidad curricular, los aportes de los sujetos participantes de la investigación reflejan una coincidencia con Clavijo y Bocanumen Gutiérrez (2006), quienes señalan que los módulos permiten el paso de una instrucción centrada en el profesorado, a una construcción y descubrimiento centrado en el estudiantado.

Además, las metodologías y evaluaciones aplicadas dentro de los cursos modulares promueven un aprendizaje para la vida, por cuanto las experiencias buscan, con las estrategias didácticas, ser significativas $y$, de esta manera, permiten un aprendizaje personalizado, de acuerdo con los intereses y necesidades de cada estudiante, desde un eje problematizador (García Fallas et al., 2014).

Sin embargo, tener la variedad de metodologías, las cuales pueden lograr ese aprendizaje significativo; pero sin la comprensión, por parte del personal docente, sobre el alcance que tienen estas, no solo dentro de la flexibilidad, sino más importante aún, en el estudiantado, resulta en praxis pedagógicas descontextualizadas, tradicionalistas, centradas en el personal docente, y un fracaso en el alcance del objetivo pedagógico, el cual consiste en que el estudiantado aprenda.

\section{Conclusiones}

La investigación arroja que la flexibilidad curricular no dispone de una definición global, ni siquiera a nivel local entre las mismas universidades estatales. Las referencias consultadas son claras en que tanto los términos, los conceptos y las transformaciones son propias de cada espacio, a pesar de responder a tendencias mundiales.

La flexibilidad curricular no ha tenido una trayectoria vertical descendente, desde lo internacional hacia lo nacional dentro de las universidades, y de estas hacia las unidades académicas. En la UCR, han sido las mismas escuelas y sedes las instancias que han partido de lo internacional para implementar, en sus actividades, sistemas flexibles dentro del proceso didáctico. Es esta secuencia la que permitió a esta institución ver la necesidad de normar la flexibilidad curricular, lo que explica que primero se dieran este tipo de cambios dentro de la carrera GERENA (creada en 2009).

A pesar de los años de emitida la normativa institucional VD-R-9374-2016, el personal docente de la carrera no identifica la plataforma Mediación Virtual como oficial, refieren porcentajes de virtualidad en sus cursos, pero señalan herramientas ajenas a la institución 
http://doi.org/10.15359/ree.25-2.13

http://www.una.ac.cr/educare

educare@una.ac.cr

como Dropbox, WhatsApp, Drive, entre otros. Asimismo, expresan que los componentes de los programas se presentan desde un enfoque positivista, como objetivos o contenidos, perspectiva congruente con lo manifestado por el estudiantado, tanto en metodologías como en evaluación, y no existe una coordinación académica entre el profesorado a cargo de los módulos. Este aporte del estudio resalta lo expuesto en las posiciones teóricas, debido a que se potencian los aspectos logísticos, pero se desvinculan, del valor real, los aspectos pedagógicos.

Con los hallazgos de esta investigación es posible determinar que las unidades académicas han logrado la trascendencia a una docencia innovadora, buscando la intercomunicación que no se señala en la definición institucional (de la UCR), pero que se ha ido construyendo de manera colectiva, a partir de las necesidades y realidades surgidas en los contextos de la educación superior actual.

En la evaluación de los dos cursos modulares de la carrera GERENA, se ha encontrado en la praxis modelos más flexibles, con un impulso del enfoque constructivista, el cual se promueve desde organismos internacionales y los cambios responden a las tendencias mundiales. Sus estrategias metodológicas coinciden con este enfoque, basado en la resolución de problemas, el aprendizaje significativo y el desarrollo de habilidades y destrezas. Se reitera, con este elemento concluyente, los referentes conceptuales que señalaban la existencia de proyectos piloto dentro de las tendencias de la flexibilidad, pero sin cambios generalizados en los métodos pedagógicos.

La Universidad de Costa Rica requiere divulgar sus propias tendencias o concepciones respecto de la flexibilidad, así como una reflexión o discusión de los alcances de esta dentro de la praxis pedagógica, lo cual permita que el personal docente siga implementando esos sistemas flexibles (como el uso de las TIC), pero reconociendo el fundamento que la universidad establece para tales fines.

\section{Declaración de Material complementario}

Este artículo tiene disponible, como material complementario:

-La versión preprint del artículo en https://doi.org/10.5281/zenodo.4042040

\section{Referencias}

Bautista, G., Borges, F. y Forés, A. (2006). Didáctica universitaria en entornos virtuales de enseñanzaaprendizaje. Narcea. http://www.terras.edu.ar/biblioteca/2/2BAUTISTA-GuillermoBORGES-Federico-FORES-AnnaCAP2Ser-estudiantes-en-entornos-virtuales.pdf

Camacho Zúñiga, M. G., Lara Alemán, Y. y Sandoval Díaz, G. (2016). Diseño curricular para entornos virtuales de aprendizaje en la Universidad Técnica Nacional, Costa Rica. Área de Tecnología Educativa y Producción de Recursos Didácticos, Universidad Técnica Nacional. https:// recursos.portaleducoas.org/sites/default/files/VE16.754.pdf 
http://doi.org/10.15359/ree.25-2.13

http://www.una.ac.cr/educare educare@una.ac.cr

Cervantes-Pérez, F., Vadillo, G., Bucio, J. y Herrera, A. (2019). Characterizing UNAM's open education system using the OOFAT model. International Review of Research in Open and Distance Learning, 20(4), 212-230. https://doi.org/10.19173/irrodl.v20i3.4108

Cisterna Cabrera, F. (2005). Categorización y triangulación como procesos de validación del conocimiento en una investigación cualitativa. Theoria, 14(1), 61-71. https://www.redalyc. org/pdf/299/29900107.pdf

Clavijo, O. E. y Bocanumen Gutiérrez, T. (2006). El módulo como herramienta de aprendizaje en el proceso docente educativo en la modalidad semipresencial y cursos intensivos en las regiones [Trabajo de grado]. Universidad de Antioquia, Colombia, Medellín. http://ayura.udea.edu. co:8080/jspui/handle/123456789/291

Collis, B. y Moonen, J. (2011). Flexibilidad en la educación superior: Revisión de expectativas. Comunicar, 19(37)), 15-25. http://dx.doi.org/10.3916/C37-2011-02-01

Consejo Nacional de Rectores. Oficina de Planificación de la Educación Superior. (2016). Plan Nacional de la Educación Superior Universitaria Estatal 2016-2020. Autor. https://www. conare.ac.cr/images/articulos/planes_2016_2020.pdf

Flannery, M. y McGarr, O. (2014). Flexibility in higher education: An irish perspective. Irish Educational Studies, 33(4), 419-434. http://dx.doi.org/10.1080/03323315.2014.978658

García Fallas, J., Guzmán Aguilar, A. y Murillo Sancho, G. (2014). Evaluación de competencias y módulos en un currículo innovador El caso de la licenciatura en Diseño y Desarrollo de Espacios Educativos con TIC de la Universidad de Costa Rica. Perfiles Educativos, 26(143), 67-85. https://doi.org/10.1016/S0185-2698(14)70610-5

Hidalgo, G. (2001). Hacia un perfil de Universidad a Distancia para la UNED de Costa Rica. Revista Iberoamericana de Educación a Distancia, 4(2), 71-98. https://doi.org/10.5944/ried.4.2.1180

Jiménez Castro, L. M. (2008). Enfoque curricular centrado en la persona. Revista Educación 32(1), 63-76. Costa Rica. https://doi.org/10.15517/REVEDU.V3211.524

Lira Valdivia, R. I. y Fëdorov Fëdorov, A. (2002). El aprendizaje en-línea: Una experiencia en el Instituto Tecnológico de Costa Rica. Revista Educación, 26(2), 197-212. https://doi. org/10.15517/REVEDU.V26I2.2916

Mirriahi, N., Alonzo, D y Fox, B. (2015). A blended learning framework for curriculum design and professional development. Research in Learning Technology, 23, 1-14. https://doi. org/10.3402/rlt.v23.28451

Palmer, S. R. (2011). The lived experience of flexible education - theory, policy and practice. Journal of University Teaching \& Learning Practice, 8(3), 1-14. http://ro.uow.edu.au/jutlp/ vol8/iss $3 / 2$ 
http://doi.org/10.15359/ree.25-2.13

http://www.una.ac.cr/educare

educare@una.ac.cr

Pedroza Flores, R. (2004). La flexibilidad académica en la universidad pública. Revista de la Educación Superior, 119, 1-15. http://publicaciones.anuies.mx/pdfs/revista/Revista119 S3A1ES.pdf

Pedroza Flores, R. y García Briceño, B. (2005). Flexibilidad académica y curricular en las instituciones de educación superior. Miguel Ángel Porrúa.

Tourón, J. y Campión, R. S. (2013). Atención a la diversidad y desarrollo del talento en el aula. El modelo DT-PI y las tecnologías en la implantación de la flexibilidad curricular y el aprendizaje al propio ritmo. Revista Española de Pedagogía, 71(256), 441-459. https:// revistadepedagogia.org/wp-content/uploads/2013/09/256-03.pdf

Universidad de Costa Rica, Consejo Universitario. (05 de junio, 2015). Políticas de la Universidad de Costa Rica 2016-2020. Excelencia e innovación con transparencia y equidad. La Gaceta Universitaria, 13, pp. 1-7. http://www.cu.ucr.ac.cr/uploads/tx ucruniversitycouncildatabases/normative/politicas institucionales 2016-2020.pdf

Universidad Nacional, Consejo Universitario. (31 de octubre, 2012). Reglamento o modificaciones a reglamentos. Gaceta extraordinaria, 21, pp. 1-120. http://www.documentos.una.ac.cr/ bitstream/handle/unadocs/6092/Ajuste\%20del\%20ciclo\%20lectivo\%20de\%2018\%20 a\%2017\%20semanas.pdf?sequence $=1$ \&isAllowed $=y$

Universidad de Costa Rica, Vicerrectoría de Docencia. (2016a). Resolución VD-R-9374-2016: Marco de Referencia para el Desarrollo de la Docencia en Entornos Virtuales en la Universidad de Costa Rica. http://vd.ucr.ac.cr/documento/vd-r-9374-2016/

Universidad de Costa Rica, Vicerrectoría de Docencia. (2016b). Resolución VD-R-9391-2016. Creación del espacio de docencia multi-, inter-, y transdiciplinario (EDOMIT). http://vd.ucr. ac.cr/documento/vd-r-9391-2016/

Zawacki-Richter, O., Conrad, D., Bozkurt, A., Aydin, C. H., Bedenlier, S., Jung, I., Stöter, J., Veletsianos, G., Blaschke, L. M., Bond, M., Broens, A., Bruhn, E., Dolch, C., Kalz, M., Kerres, M., Kondakci, Y., Marin, V., Mayrberger, K., Müskens, W., Naidu, S., Qayyum, A., Roberts, J., Sangrà, A., Loglo, F. S., Slagter van Tryon, P. J. y Xiao, J. (2020). Elements of open education: An invitation to fture research. International Review of Research in Open and Distance Learning, 21(3), 319334. 10.19173/irrodl.v21i3.4659 